UCRL-JC-130019

PREPRINT

\title{
High Gain X-ray Lasers Pumped by Transient Collisional Excitation
}

\section{J. Dunn, A. L. Osterheld, V. N. Shlyaptsev, J. R. Hunter,} R. Shepherd, R. E. Stewart, W. E. White

This paper was prepared for submittal to the 11th American Physical Society Topical Conference on

Atomic Processes in Plasmas

Auburn, AL

March 22-26, 1998

June 16, 1998

This is a preprint of a paper intended for publication in a journal or proceedings. Since changes may be made before publication, this preprint is made available with the understanding that it will not be cited or reproduced without the permission of the author. 


\section{DISCLAIMER}

This document was prepared as an account of work sponsored by an agency of the United States Government. Neither the United States Government nor the University of California nor any of their employees, makes any warranty, express or implied, or assumes any legal liability or responsibility for the accuracy, completeness, or usefulness of any information, apparatus, product, or process disclosed, or represents that its use would not infringe privately owned rights. Reference herein to any specific commercial product, process, or service by trade name, trademark, manufacturer, or otherwise, does not necessarily constitute or imply its endorsement, recommendation, or favoring by the United States Government or the University of California. The views and opinions of authors expressed herein do not necessarily state or reflect those of the United States Government or the University of California, and shall not be used for advertising or product endorsement purposes. 


\title{
High Gain X-ray Lasers Pumped by Transient Collisional Excitation
}

\author{
J. Dunn, A.L. Osterheld, V.N. Shlyaptsev ${ }^{\dagger}$, J.R. Hunter, \\ R. Shepherd, R.E. Stewart, and W.E. White \\ Lawrence Livermore National Laboratory, P.O. Box 808, Livermore, CA 94551 \\ Permanent Address: P.N. Lebedev Physical Institute, 117924 Moscow, Russia.
}

\begin{abstract}
We present recent results of x-ray laser amplification of spontaneous emission in Ne-like and Ni-like transient collisional excitation schemes. The plasma formation, ionization and collisional excitation can be optimized using two laser pulses of $1 \mathrm{~ns}$ and 1 ps duration at table-top energies of $5 \mathrm{~J}$ in each beam. High gain of $35 \mathrm{~cm}^{-1}$ has been measured on the $147 \AA 4 \mathrm{~d} \rightarrow 4 \mathrm{p} \mathrm{J}=0 \rightarrow 1$ transition of Ni-like Pd and is a direct consequence of the nonstationary population inversion produced by the high intensity picosecond pulse. We report the dependence of the x-ray laser line intensity on the laser plasma conditions and compare the experimental measurements with hydrodynamic and atomic kinetics simulations for $\mathrm{Ne}$-like and Ni-like lasing.
\end{abstract}

\section{INTRODUCTION}

The first laboratory demonstration of x-ray laser amplification of spontaneous emission was achieved in 1984 by pumping with high power laser drivers $(1,2)$. Since that time different $x$-ray laser schemes working on a range of ions, as described in recent proceedings $(3,4)$, have been investigated. In particular, progress has been rapid in small scale table-top x-ray lasers in the last three to four years. In the work by Rocca et al., the fast capillary discharge collisional x-ray laser was produced on the $3 p \rightarrow 3 s J=0 \rightarrow 1$ transition of Ne-like Ar at $469 \AA$ (5) and later the output was increased to the saturation limit (6). The development of compact, high power subpicosecond lasers based on chirped pulse amplification (CPA) (7) has also been very important for extending X-ray lasers to shorter wavelengths. This is because the pumping power required to produce the plasma formation, ionization and excitation for shorter wavelength collisional $\mathrm{x}$-ray lasers has traditionally scaled approximately as $\lambda^{-4}$, where $\lambda$ is the $\mathrm{x}$-ray laser wavelength.

Short pulse, high power laser drivers at the Terawatt level or higher with high repetition rates can provide the necessary pump for a new class of x-ray laser (XRL). A novel scheme was reported in 1995 for a Pd-like Xe x-ray laser 
operating at $10 \mathrm{~Hz}$ with a gain length product of $g L \sim 11$ at $418 \AA$ for $40 \mathrm{fs}$ irradiation of a xenon gas cell (8). This scheme used a combination of field induced tunneling ionization followed by collisional excitation. A shorter wavelength scheme operating at $135 \AA$ with a gain length product of $g L \sim 6$ has also been demonstrated for an H-like Li Ly- $\alpha$ inversion pumped by a 300 fs laser pulse (9) in a micro-capillary. The transient collisional excitation using a picosecond pulse has been reported recently by Nickles et al. for the $3 p \rightarrow 3 s J=0 \rightarrow 1$ transition of Ne-like Ti which lases at $326 \AA$ with a gain of $19 \mathrm{~cm}^{-1}$ and a gain length product of $g L$ $\sim 9.5$ (10). This scheme has also been reported to work with a $g L>14$ for Ti and at $255 \AA$ for Ne-like Fe $(11,12)$, and to achieve saturated output on Ne-like Ti and Ge at $196 \AA$ (13).

The Ni-like ion has the similar benefits of the closed shell configuration as the Ne-like ion and therefore it is a robust XRL plasma medium. It also has the advantage of a larger x-ray photon energy to excitation energy ratio: the ratio is $\sim 0.19$ for Ni-like palladium. Therefore, while the Ne-like transient collisional scheme is interesting, the extension to the Ni-like ion sequence yields higher efficiency, higher output and shorter wavelength x-ray lasers. Previously, Ni-like collisional x-ray lasers for $3 d^{9} 4 d \rightarrow 3 d^{9} 4 p$ transitions in high- $Z$ ions have required large energy laser drivers $(14,15)$. Using the NOVA laser, short wavelength lasing has been extended to $35 \AA$ for the $4 d \rightarrow 4 p \quad J=0 \rightarrow 1$ transition of Ni-like Au (16). Lasing on Ni-like ions has also been measured on the Lanthanide series for example Neodymium and Lanthanum for x-ray lasers operating at wavelengths of $79 \AA$ and $89 \AA$ (17). Saturated output for the Ni-like scheme has been demonstrated very recently at $140 \AA$ for $\mathrm{Ag}$ and at $73 \AA$ for Sm by using $150 \mathrm{~J}$ of energy in a 70 ps pulse and pre-pulse combination (18).

We have recently observed lasing on the transient collisional excitation Ni-like $\mathrm{Pd}$ scheme pumped with less than $10 \mathrm{~J}$ of laser energy (19). This is a further reduction in the pumping energy by more than one order of magnitude. Gain in excess of $35 \mathrm{~cm}^{-1}$ and a $g L$ product of $\sim 12.5$ have been measured on the $4 d \rightarrow 4 p$ $J=0 \rightarrow 1$ transition at $147 \AA$ for this scheme. The next section describes the transient gain scheme and the experimental implementation. Section 3. summarizes experimental results on the transient Ni-like Pd scheme. Section 4. compares the Ni-like Pd results to simulations while Section 5. discusses the important issue of $\mathrm{Ne}$-like Ti output as a function of short pulse delay. The final part of the paper, Section 6., discusses some future trends including a brief description of the purpose built table-top COMET laser driver for the next phase of experiments.

\section{TRANSIENT GAIN SCHEME DESCRIPTION AND EXPERIMENTAL IMPLEMENTATION}

The transient collisional excitation (TCE) or transient gain scheme as proposed by Afanasiev and Shlyaptsev $(20,21)$ describes the creation of a short-lived inversion on the timescale of femtoseconds to a few tens of picoseconds. This is 
characterized by a plasma lifetime dictated by the fast pumping source which in turn should be comparable with the relaxation timescales of the excited levels. Most collisional x-ray lasers reported in the last decade have been variations of the quasisteady state (QSS) inversion scheme where the pumping source is much longer than the lifetime of the excited levels. The TCE scheme differs from the QSS inversion in a number of important areas but mainly because the risetime of the level excitation rates is shorter than the collisional excitation timescales. This produces a short-lived transient population inversion pumped directly from the ground state until collisions redistribute the populations among all levels achieving finally the quasi-steady state. During the time of population redistribution the inversion is not defined by the small difference in populations of upper and lower level as in the case of QSS but in fact solely by the the upper laser level population. Besides, since the plasma can be made sufficiently hot for maximal level population during this short transient time it is predicted that TCE will produce very high gains above $100 \mathrm{~cm}^{-1}$ and therefore very high efficiency $\mathrm{x}$-ray lasers.

One proposed method for demonstrating the transient scheme $\mathrm{x}$-ray laser is to use two sequential stages of laser irradiation $(10,21)$. A formation pulse of $1 \mathrm{~ns}$ heats a solid planar target at $10^{12} \mathrm{~W} \mathrm{~cm}^{-2}$ to produce a long scalelength plasma with a high fraction of ions in the Ni-like stage. It is essential for a delay before the second pulse to optimize the conditions within the plasma for maximum amplification. The short $1 \mathrm{ps}$ pump pulse at $10^{15} \mathrm{~W} \mathrm{~cm}^{-2}$ produces rapid plasma heating with an increase in the electron temperature $T_{e} \geq \Delta E_{e}$, where $\Delta E_{e}$ is the upper laser level excitation energy. This generates the transient inversion and the scheme works most efficiently when the plasma formation conditions are at the correct ionization, with low initial electron temperature and low electron density gradients. The high gain conditions last for a few picoseconds then will quickly decay after $\sim 10 \mathrm{ps}$ as a result of collisional redistribution of the electron population among all excited levels, ionization and plasma cooling. Therefore, high gain is expected for short 0.1 to $0.3 \mathrm{~cm}$ targets with decreasing gain for longer lengths.

The Ni-like Pd experiments were performed at the Lawrence Livermore National Laboratory JANUS laser facilities. One arm of the JANUS laser provided an $800 \mathrm{ps}$ (FWHM) pulse at $1064 \mathrm{~nm}$ wavelength with $5-6 \mathrm{~J}$ on target at a repetition rate of 1 shot/ 3 minutes. This produced the plasma forming beam. The short pulse needed to pump the inversion was provided by the $5-6 \mathrm{~J}$ hybrid chirped pulse amplification JANUS $500 \mathrm{fs}$ system. This is based on a Ti:Sapphire oscillator and regenerative amplifier front end tuned to $1053 \mathrm{~nm}$ wavelength with Nd:phosphate glass power amplifiers. The pulse duration was lengthened to $1.1 \mathrm{ps}$ (FWHM) by de-tuning the compressor gratings. The regenerative amplifiers of the two lasers were synchronized using the $80 \mathrm{Mhz}$ radio-frequency output from the short pulse oscillator resulting in a relative timing jitter of $80 \mathrm{ps}$ rms. The arrival of the short pulse was delayed by 1 to $2 \mathrm{~ns}$ relative to the peak of the long pulse to minimize refraction effects and to allow for sufficient plasma cooling. After amplification, the beams were enlarged to $8.4 \mathrm{~cm}$ diameter, aligned and co-propagated under vacuum 
to the target chamber. The combination of a long focus cylindrical lens and a paraboloid were used to produce a line focus of dimensions $70 \mu \mathrm{m} \times 12.5 \mathrm{~mm}$. Slab palladium targets were used in the experiment. A flat-field grating spectrometer with a back-thinned CCD detector measured the axial spectral emission. Further experimental details are described in $(11,19)$.

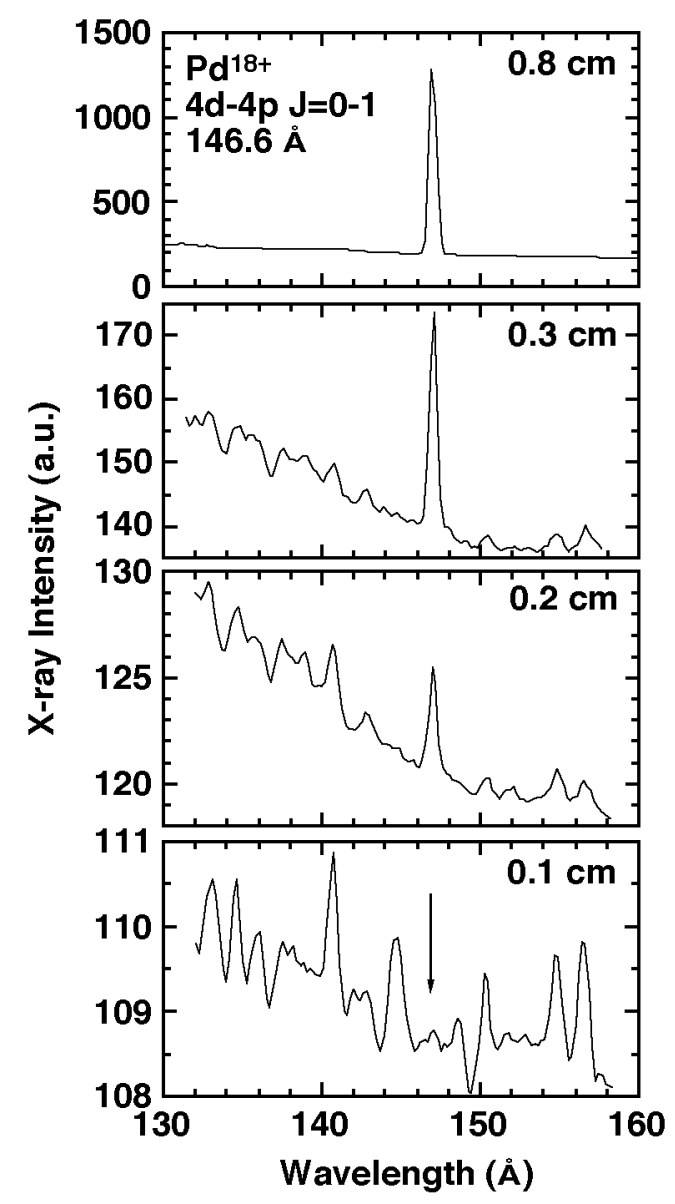

FIGURE 1. Axial spectra for various target lengths of $P d$, from 0.1 to $0.8 \mathrm{~cm}$, showing the exponential increase in the Ni-like $\mathrm{Pd} 4 d \rightarrow 4 p \mathrm{~J}=0 \rightarrow 1 \mathrm{x}$-ray laser line at $146.6 \AA$ measured in second order. The transition, weak but visible, is arrowed in the $1 \mathrm{~mm}$ target length, bottom panel. The laser line experiences 4 orders of magnitude increase for a $0.8 \mathrm{~cm}$ target. Note the change in the intensity scale for each panel: there is a factor of 500 difference in the intensity scale between the bottom panel and top panel.

\section{EXPERIMENTAL RESULTS}

Figure 1 shows the axial spectrum for different target lengths. The strong exponential increase in the $4 d \rightarrow 4 p J=0 \rightarrow 1$ transition is observed unambiguously in 
second order. Incident laser energy on target was approximately $4 \mathrm{~J}$ of long pulse and $5 \mathrm{~J}$ of short pulse. The lasing wavelength is measured to be $146.6 \pm 0.9 \AA$ by fitting a high order polynomial function to the spectrum using Ne-like transient gain $3 \rightarrow 3$ x-ray laser lines of $\mathrm{Ti}$ and $\mathrm{Fe}$, observed in first order, as calibration lines. These Ne-like lasing lines have been previously reported in long pulse collisional excitation schemes using the pre-pulse technique (22). The Ni-like Pd wavelength is in good agreement with the calculated wavelength of $148 \AA$ (23). (More accurate wavelengths have been recently measured and extended to other ions on the Ni-like isoelectronic sequence (24) using the table-top COMET laser driver.) As can be seen from Fig. 1 the output of the line increases rapidly with small increments of target length. The laser dominates the spectrum above $0.3 \mathrm{~cm}$ targets. The largest increases in $x$-ray laser output are observed for 0.05 and $0.1 \mathrm{~cm}$ steps in the shortest targets.

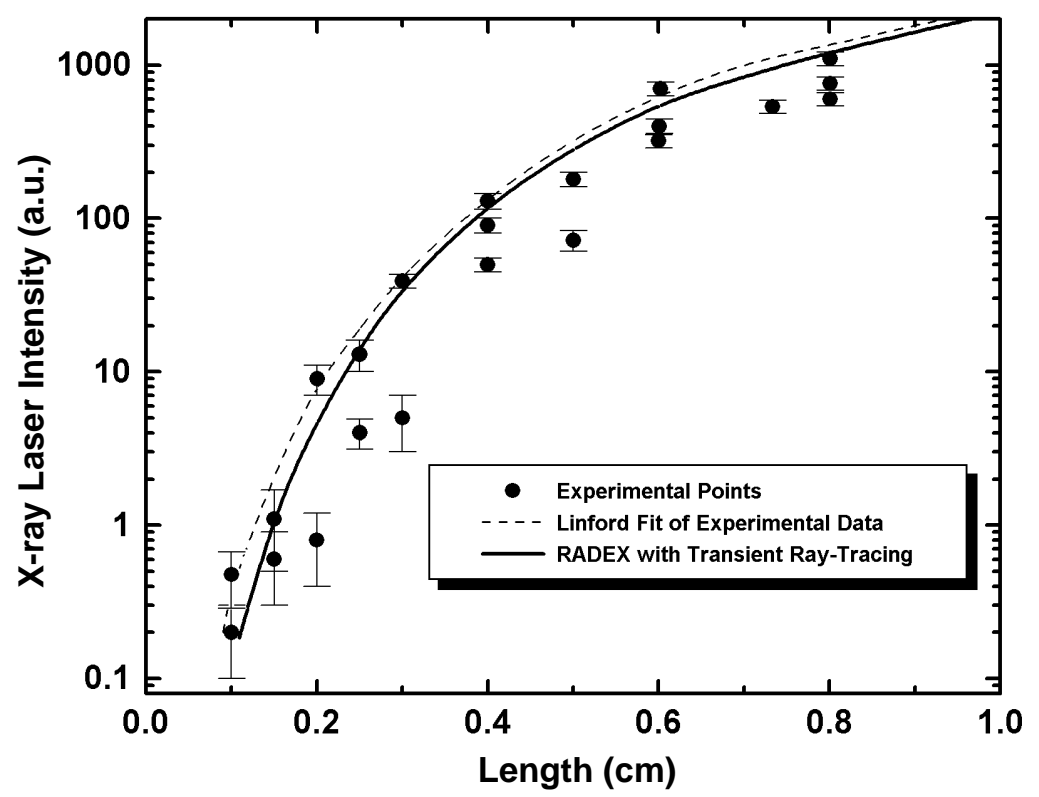

FIGURE 2. Intensity of $146.6 \AA$ laser line, measured in second order, for 0.1 to $0.8 \mathrm{~cm} \mathrm{Pd}$ target lengths. Full circles are experimental data. Dashed curve is Linford fit to experimental data. Solid curve is RADEX simulations with transient gain ray-tracing.

Figure 2 plots the measured laser line intensity as a function of target length. More than four orders of magnitude increase in the laser output is observed when the target is lengthened from 0.1 to $0.8 \mathrm{~cm}$. All laser driver conditions, including the short pulse energy, long pulse energy, line focus and delay between the two pulses, are kept constant. Also shown are the local Linford fit to the experimental points (25) and the RADEX simulations with transient ray-tracing. The latter is discussed in more detail in the next section. The shape of the intensity versus length output of the x-ray laser indicates continually changing gain conditions with the 
highest gain of $35 \mathrm{~cm}^{-1}$ observed at the shortest target lengths of 0.1 to $0.2 \mathrm{~cm}$. The gain drops at intermediate and longer target lengths. Although the shape is similar to saturation, this effect is explained by the transient gain timescale lasting for 5 to $15 \mathrm{ps}$. This is significantly shorter than the x-ray laser propagation time along the line focus (26) and so the laser experiences continually decaying gain conditions as it travels along the gain medium. The overall $g L$ product is determined to be $\sim 12.5$ by integrating the measured gain along the full target length. The hydrodynamics, atomic kinetics modeling and ray-tracing are described in the next section.

\section{COMPARISON OF NI-LIKE PALLADIUM RESULTS WITH SIMULATIONS}

Very high gains in excess of $300 \mathrm{~cm}^{-1}$ have been predicted for transient gain in Ni-like Xe at $96 \AA$ (21) and for Ni-like Mo $189 \AA$ (27) at laser irradiances and pulse durations close to the work described in (19). High values have also been predicted for transient gain Ne-like schemes (28). The experimental gain of $35 \mathrm{~cm}^{-1}$ measured for Pd is high in comparison with previously reported $\mathrm{x}$-ray gain results but still significantly lower than the predictions. The main reasons for the observed lower gain are the combined effects of refraction deflecting the x-ray laser out of the gain region of the plasma, short-lived high gain and collisional line broadening. We used the 1-dimensional numerical code $\operatorname{RADEX}(10,20,21)$ which treats the transient hydrodynamics, atomic kinetics and radiation transport self-consistently. An additional ray-tracing package, as a post-processor, is used to model the propagation of the $\mathrm{x}$-ray laser along the gain medium and calculate the $\mathrm{x}$-ray laser intensity. It is important to note that the hydrodynamics, atomic kinetics and raytracing have to be made using a transient approximation to simulate the experimental conditions. In particular, calculations show that if the x-ray laser line ray-tracing is made in a quasi-steady state approximation for gain described as transient this produces results inconsistent with the observed $\mathrm{x}$-ray laser characteristics including the lasing intensity, pulse duration, deflection angle and effective gain. This can be explained mainly by the fast gain risetime of $1-3 \mathrm{ps}$ and short lifetime of $5-15 \mathrm{ps}$ (for plasma densities $n_{e} \sim 1-3 \times 10^{20} \mathrm{~cm}^{-3}$ ) compared to the propagation time $L / c \sim 30 \mathrm{ps}$ along the amplified medium. The major observed XRL characteristics are consistent with the experiment only when the effects of fast temporal evolution of the gain and photon transit time are included and properly described.

Figure 3. shows output from RADEX for simulations of the experimental conditions used to generate the Ni-like $\mathrm{Pd}$ X-ray laser mainly during or after the laser short pulse which produces the transient excitation. A general overview is that during the short pulse laser, the electron temperature exhibits dramatic changes near the critical density where locally most of the absorbed laser energy is deposited. The transient gain here reaches ultra-high values during the short pulse but also 
vanishes very rapidly. Two other regions, the over- and under-critical, also produce high gain: the former is on the front of the strong heat conduction wave. The latter, where much of the observed XRL gain is measured, is in the region of inverse bremsstrahlung absorption where the increase in the temperature is sufficiently high for efficient transient excitation $T_{e} \sim \Delta E_{e}$, where $\Delta E_{e}$ is the excitation energy.

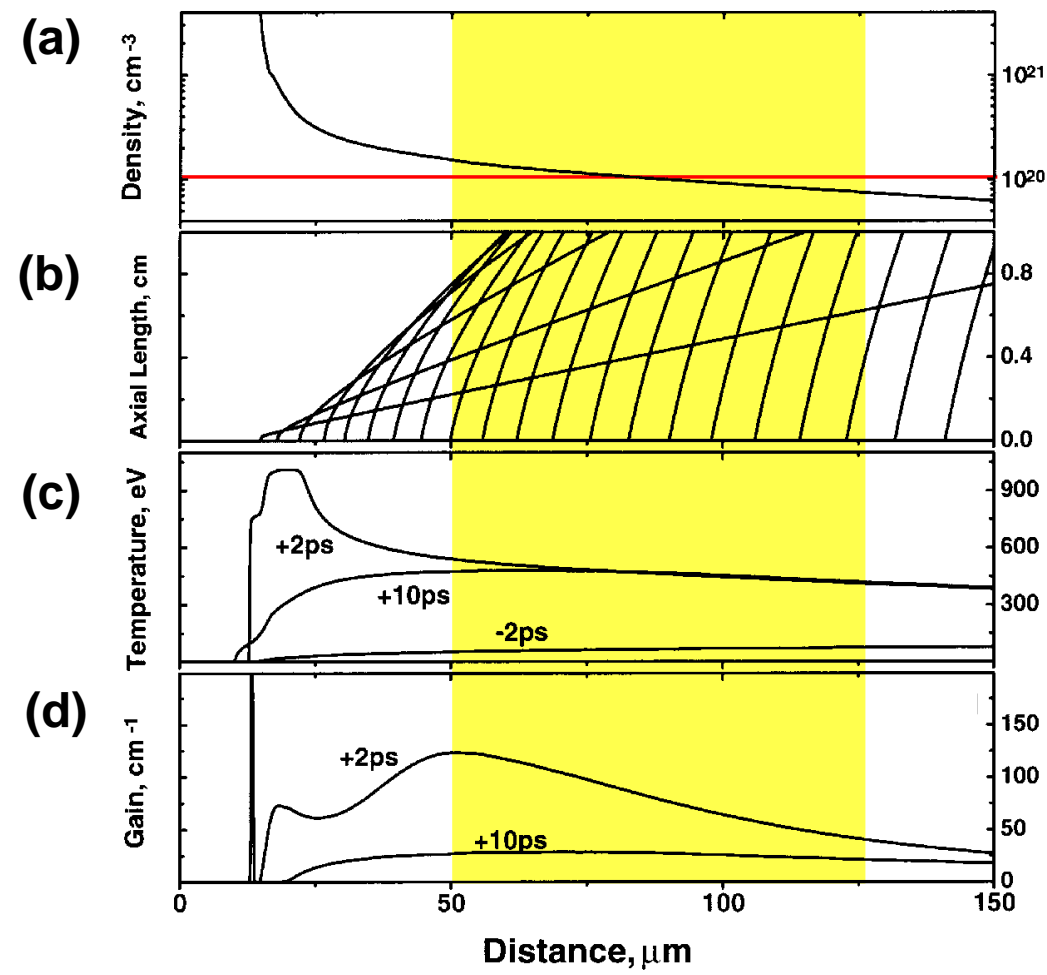

FIGURE 3. Simulations from the one-dimensional numerical code RADEX of the experimental lasing conditions for the Ni-like $\mathrm{Pd} 4 d \rightarrow 4 p \mathrm{~J}=0 \rightarrow 1$ transition at $147 \AA$. The plasma formation pulse of 850 ps (FWHM) duration arrives $1.34 \mathrm{~ns}$ before the peak of the $1.1 \mathrm{ps}$ short pulse. The conditions above are relative to the peak of the short pulse. The shaded area between 50 and 125 $\mu \mathrm{m}$ is region where strongest lasing is observed but is not confined to this region only. (a) Electron density profile at 2 ps after the peak of the short pulse laser. Horizontal line indicates $0.1 n_{c}$ position, $10^{20} \mathrm{~cm}^{-3}$. (b) Ray-tracing of $x$-ray laser beam for target lengths up to $1 \mathrm{~cm}$ at $2 \mathrm{ps}$ after peak of short pulse laser. (c) Electron temperature profile for -2 ps (-2 ps curve), +2 ps (+2 ps curve) and +10 ps relative to peak of short pulse. (d) Gain profile observed at $+2 \mathrm{ps}$ and $+10 \mathrm{ps}$.

Looking at Fig. 3 in more detail, it is observed that refraction is caused by density gradients normal to the target surface, as shown in Fig. 3 (a) for +2 ps after the peak of the picosecond pulse. These deflect the laser out of the high density and high gain region as it propagates along the plasma column. Critical density is $\sim 15 \mu \mathrm{m}$ in front of the target. This is clearly shown for the XRL trajectory plot of 
Fig. 3 (b) at +2 ps, where refraction stops amplification in the high gain region at $n_{e}$ $\sim 10^{21} \mathrm{~cm}^{-3}$ for plasma lengths $L<0.1-0.3 \mathrm{~cm}$. This indicates that refraction is still very important for the shorter $147 \AA$ wavelength here than in the transient Ne-like Ti x-ray laser at $326 \AA$ [10]. It is also applicable to the shorter target lengths of $0.8 \mathrm{~cm}$ than in the few centimeter long geometries QSS schemes. However, refraction is much less pronounced at lower densities $n_{e} \sim 1-3 \times 10^{20} \mathrm{~cm}^{-3}$ for $147 \AA$ photons of Ni-like Pd compared to $326 \AA$ Ne-like Ti x-ray laser.

Secondly, the conjunction of the fast transient nature of the atomic kinetics and the finite photon transit time $L / C$ is significant. This transit time effect, when the XRL experiences gradually decreasing gain during propagation evident in Fig. 2, is basically not observed in most QSS lasers. This is illustrated in Fig. 3 (c) for the predicted temperature distribution in space at three different times. The long plasma formation pulse heats the plasma to a peak temperature of $T_{e} \sim 150-200 \mathrm{eV}$ sufficient to ionize Pd to the Ni-like charge stage. At the end of the long pulse, the plasma expands and cools to less than $\sim 90 \mathrm{eV}$ as shown by the -2 ps curve, representing 2 ps prior to the short pulse arrival, without significant change in the Ni-like abundance. The absorption of the picosecond pulse energy occurs mostly at the critical density surface at $n_{e}=10^{21} \mathrm{~cm}^{-3}$ rapidly heating the electron temperature there to $\sim 2 \mathrm{keV}$ in the first few picoseconds, curve $+2 \mathrm{ps}$. There is partial heating in the under-dense corona to an optimal of $350-500 \mathrm{eV}$. As a result of strong heat flux, close to the free streaming limit, the high temperature region cools to $400 \mathrm{eV}$ after $10 \mathrm{ps}$ (curve $+10 \mathrm{ps}$ ), and then continues to fall slowly. The transient $J=0 \rightarrow 1$ gain, Fig. 3 (d), reaches high values of $200 \mathrm{~cm}^{-1}$ during the first $1-3 \mathrm{ps}$ and $50-$ $80 \mathrm{~cm}^{-1}$ during a further $10 \mathrm{ps}$. The former occur near the critical density in the thin ablative layer, the isolated spike in Fig. 3 (d) at $15 \mu \mathrm{m}$, while the latter are in areas of relatively flat lower density profile, shaded region. As indicated by Fig. 3 (b) and (d), the short inversion lifetime near the high density critical region decreases the photon transit path in the high gain region to an axial length of $\sim 500 \mu \mathrm{m}$.

The third important phenomenon is the dominance of collisional line broadening over Doppler broadening of the excited levels at electron densities above $0.3 \times$ critical. As a consequence of all these effects, the gain-length product at high density and gain region is substantially decreased. The outer plasma regions at lower density $n_{e} \sim 0.9-2 \times 10^{20} \mathrm{~cm}^{-3}$ are more optimal for amplification resulting in larger local $g L$. Hence, the unusual properties of the TCE scheme such as the high gain and saturation-like behaviour find consistent explanation by a combination of the above main plasma and transient kinetics effects. Thus, returning to Fig. 2 the saturation-like behavior for the intensity of Ni-like Pd x-ray laser with length obtained in the experiment, is well reproduced in the RADEX calculations. The dashed curve on this figure represents the Linford fit to the experimental points at highest intensity for a given length. These estimations and numerical investigations indicate that x-ray laser intensity saturation is in fact very close and can be achieved by several methods including just simply increasing the 
plasma length. Calculations also suggest that the high efficiency obtained in this work can be further improved with the use of a pre-pulse, low density targets or traveling wave irradiation [21].

\section{NE-LIKE TI LASING FOR DELAYED SHORT PULSE}

An explanation of the long pulse-to-short pulse delay was one of the important questions to be answered for effective laser operation in the transient regime. This was resolved in the current experiments. It was found experimentally for $\mathrm{Ne}$-like $\mathrm{Ti}$ $\mathrm{x}$-ray laser plasmas that lasing action had a maximum intensity if the two pulses were separated by between one and two nanoseconds (12). This issue was known but not investigated systematically in the experiments of other groups and required better understanding. Therefore, numerical modeling of this Ne-like laser scheme represented a way to explaining the optimum lasing, the strong lasing at $\sim 1.6 \mathrm{~ns}$ delay and the role of the underlying key processes.

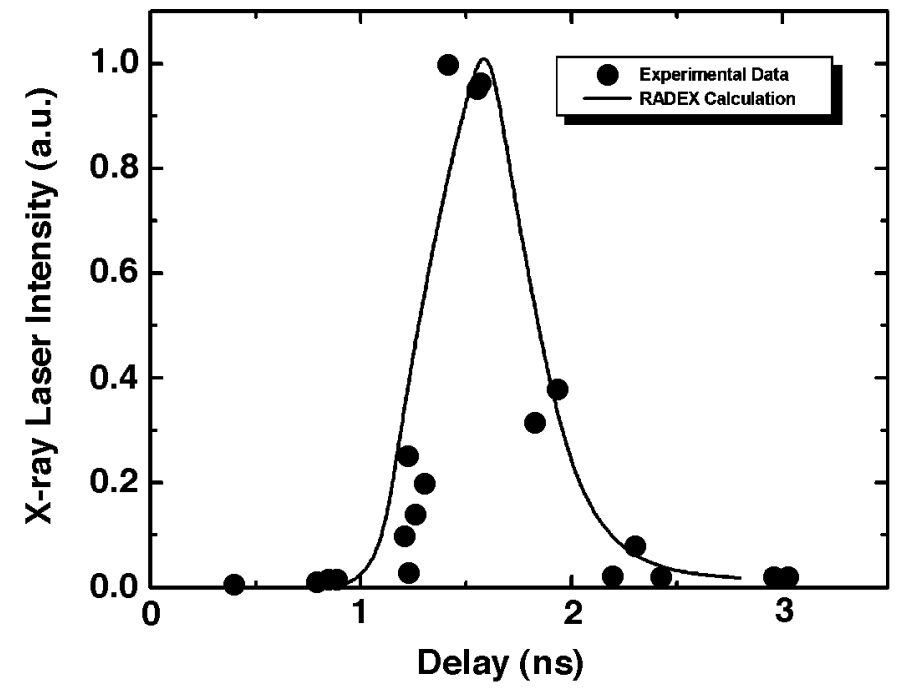

FIGURE 4. Experimental measurements of the $326 \AA$ Ne-like $\mathrm{Ti} 3 p \rightarrow 3 s$ $J=0 \rightarrow 1$ x-ray laser intensity (full circles) as a function of delayed arrival of the short pulse relative to the peak long pulse. Strong lasing is observed at a delay of $1.6 \mathrm{~ns}$. Full curve shows comparison from the one-dimensional numerical code RADEX for the experimental lasing conditions.

There exists a similarity here with previous experiments for pre-pulse formed $\mathrm{x}$ ray laser plasmas in the quasi-steady state regime. It was found in many experiments mostly conducted by Nilsen and co-workers, see for example (22), that the QSS lasing could be maximized with different kinds of low density prepulses, multiple pulses of different duration, with optimal energies and delays. The main conclusion was that enhanced laser action was a result of substantial 
suppression of refraction. With the transient excitation scheme there are several new aspects in the fundamental operation and hence the optimized parameters for it are different from the previous QSS approach. A number of comparisons have been made for the experimental data and the code RADEX simulations at the same conditions for different atomic elements.

Fig. 4 shows the intensity of Ne-like Ti $3 p \rightarrow 3 s J=0 \rightarrow 1$ laser at $326 \AA$ as a function of delay introduced for the short pulse relative to the peak of the $800 \mathrm{ps}$ plasma forming pulse. It can be seen, that lasing does not occur if the picosecond pulse arrives earlier than $1.0-1.2 \mathrm{~ns}$ after the long pulse. There is a window where good lasing is observed, centered at $\sim 1.6 \mathrm{~ns}$, followed by a fast decrease for delays of more than $2.2 \mathrm{~ns}$. This non-lasing early delay behavior is more than just strong refraction effects at the beginning of expansion. This is confirmed in the simulations by artificially reducing the effects of refraction by an order of magnitude: the time of optimal delay and width of lasing window remain relatively unchanged. The additional reason why lasing does not appear prior to a specific moment lies in the physics of the transient inversion. To achieve substantial transient gain, the initial plasma temperature before the picosecond temperature jump must be low enough to empty all excited level populations. After the $800 \mathrm{ps}$ pulse has finished, the laser plasma is allowed to cool down by expansion and radiation to reach less than $80 \mathrm{eV}$ for $\mathrm{Ti}$ and less than $90 \mathrm{eV}$ for Pd. Unless these conditions are achieved the transient gain is small. The fall in XRL intensity for large delays is due to substantial plasma expansion and the resultant drop in the density. This causes in turn a decrease in the short pulse laser absorption and hence reduced plasma temperatures during the short pulse. At these late delays the density and corresponding gradients are reduced by more than $50 \%$ which is beneficial for refraction effects but is not sufficient to compensate for the lower gain coefficient.

\section{DISCUSSION AND FUTURE DIRECTIONS}

The transient gain x-ray laser scheme opens up rich and diverse opportunities for atomic physics and plasma physics research. In particular, there are plans to study energy level measurements, short pulse laser plasma interactions and subpicosecond non-stationary atomic kinetics in plasmas. There are many possible applications which can be pursued with table-top x-ray lasers but have previously required larger laser facilities to pump the inversion. These include $\mathrm{x}$-ray microscopy (29), interferometry of plasmas (30), radiography of plasmas (31), and interferometry of materials (32). X-ray laser applications are enhanced by the high repetition shot rate and inherent short pulse duration available with table-top transient gain $\mathrm{x}$-ray laser systems. However, high output of the $\mathrm{x}$-ray laser line is essential. Gains as high as $50-100 \mathrm{~cm}^{-1}$ may be achieved with careful optimization of the target and plasma conditions. 
The original CPA short pulse laser has been re-designed with some enhancements not previously available in the first experiments to meet these

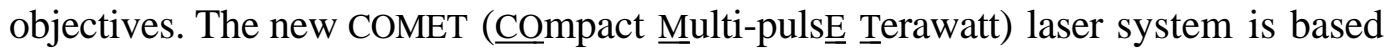
around the $15 \mathrm{TW}$ short pulse laser, maximum of $7.5 \mathrm{~J}$ in $500 \mathrm{fs}$, but has a long pulse arm sharing the same oscillator. The laser is a table-top system and occupies two standard optical tables with area less than $10 \mathrm{~m}^{2}$. The long pulse of $500-800$ ps duration has 12 - $15 \mathrm{~J}$ energy available on target and replaces the plasma forming beam previously provided by the JANUS laser. A pre-pulse network has also been installed to produce a pre-formed plasma in front of the two main pulses to improve the coupling of laser energy into the gain medium. We have recently looked at lower $\mathrm{Z}$ Ni-like materials studied previously, for example $\mathrm{Nb}$ and $\mathrm{Mo}$ (33), and observed very strong lasing on Ni-like Y through Mo from $240 \AA$ to $189 \AA$ (34). The COMET laser is a versatile system which will allow the further detailed study of the transient gain scheme.

\section{ACKNOWLEDGMENTS}

We thank B. Sellick for technical assistance. Thanks to Mark Eckart for continuing support and encouragement for this research. One of us (V.N.S.) acknowledges support from H. Baldis of ILSA and Yu. Afanasiev of LPI. This work was performed under the auspices of the U.S. Department of Energy by the Lawrence Livermore National Laboratory under Contract No. W-7405-Eng-48.

\section{REFERENCES}

1. D.L. Matthews, P.L. Hagelstein, M.D. Rosen, M.J. Eckart, N.M. Ceglio, A.U. Hazi, H. Medecki, B.J. MacGowan, J.E. Trebes, B.L. Whitten, E.M. Campbell, C.W. Hatcher, A.M. Hawryluk, R.L. Kauffman, L.D. Pleasance, G. Ramback, J.H. Scofield, G. Stone, and T.A. Weaver, Phys. Rev. Lett. 54, 106 (1985).

2. S. Suckewer, C.H. Skinner, H. Milchberg, C. Keane, and D. Voorhees, Phys. Rev. Lett. 55, 1753 (1985).

3. Proceedings of the 5th International Conference on X-ray Lasers 1996, Lund, Sweden. IOP Conf. Series No. 151, ed. S. Svanberg and C.-G. Wahlström (1996).

4. Proceedings of the conference on Soft X-ray Lasers and Applications II, ed. J.J. Rocca and L.B. Da Silva, SPIE Proceedings Vol. 3156 (1997).

5. J.J. Rocca, V.N. Shylaptsev, F.G. Tomasel, O.D. Cortázar, D. Hartshorn, and J.L.A. Chilla, Phys. Rev. Lett. 73, 2192 (1994).

6. J.J. Rocca, D.P. Clark, J.L.A. Chilla, and V.N. Shylaptsev, Phys. Rev. Lett. 77, 1476 (1996).

7. D. Strickland and G. Mourou, Opt. Commun. 56, 219 (1985).

8. B.E. Lemoff, G.Y. Yin, C.L. Gordon III, C.P.J. Barty, and S.E. Harris, Phys. Rev. Lett. 74, 1574 (1995).

9. D.V. Korobkin, C.H. Nam, S. Suckewer, and A. Goltsov, Phys. Rev. Lett. 77, 5206 (1996).

10. P.V. Nickles, V.N. Shylaptsev, M. Kalachnikov, M. Schnürer, I. Will, and W. Sandner, Phys. Rev. Lett. 78, 2748 (1997). 
11. J. Dunn, A.L. Osterheld, R. Shepherd, W.E. White, V.N. Shylaptsev, A.B. Bullock, and R.E. Stewart, SPIE Proceedings Vol. 3156, 114 (1997).

12. J. Dunn, A.L. Osterheld, R. Shepherd, W.E. White, V.N. Shylaptsev, and R.E. Stewart, Internal Report, Lawrence Livermore National Laboratory, CA UCRL-ID-127872 (1997).

13. P.V. Nickles, M. Schnürer, M.P. Kalachnikov, W. Sandner, V.N. Shylaptsev, C. Danson, D. Neely, E. Wolfrum, J. Zhang, A. Behjat, A. Demir, G. Tallents, P.J. Warwick, and C.L.S. Lewis, SPIE Proceedings Vol. 3156, 80 (1997).

14. B.J. MacGowan, S. Maxon, P.L. Hagelstein, C.J. Keane, R.A. London, D.L. Matthews, M.D. Rosen, J.H. Scofield, and D.A. Whelan, Phys. Rev. Lett. 59, 2157 (1987).

15. S. Maxon, P. Hagelstein, B. MacGowan, R. London, M. Rosen, J. Scofield, S. Dalhed, and M. Chen, Phys. Rev. A 37, 2227 (1988).

16. B.J. MacGowan, L.B. Da Silva, D.J. Fields, C.J. Keane, J.A. Koch, R.A. London, D.L. Matthews, S. Maxon, S. Mrowka, A.L. Osterheld, J.H. Scofield, G. Shimkaveg, J.E. Trebes, and R.S. Walling, Phys. Fluids B 4, 2326 (1992).

17. H. Daido, Y. Kato, K. Murai, S. Ninomiya, R. Kodama, G. Yuan, Y. Oshikane, M. Takagi, H. Takabe, and F. Koike, Phys. Rev. Lett. 75, 1074 (1995).

18. J. Zhang, A.G. MacPhee, J. Nilsen, J. Lin, T.W. Barbee Jr., C. Danson, M.H. Key, C.L.S. Lewis, D. Neely, R.M.N. O'Rourke, G.J. Pert, G.J. Tallents, J.S. Wark, and E. Wolfrum, Phys. Rev. Lett. 78, 3856 (1997); J. Zhang, A.G. MacPhee, J. Lin, E. Wolfrum, R. Smith, C. Danson, M.H. Key, C.L.S. Lewis, D. Neely, J. Nilsen, G.J. Pert, G.J. Tallents, and J.S. Wark, Science 276, 1097 (1997).

19. J. Dunn, A.L. Osterheld, R. Shepherd, W.E. White, V.N. Shylaptsev, and R.E. Stewart, Phys. Rev. Lett. 80, 2825 (1998).

20. Yu.V. Afanasiev and V.N. Shylaptsev, Sov. J. Quant. Electron. 19, 1606 (1989).

21. V.N. Shylaptsev, P.V. Nickles, T. Schlegel, M.P. Kalashnikov, and A.L. Osterheld, SPIE Proceedings Vol. 2012, 111 (1993).

22. J. Nilsen, B.J. MacGowan, L.B. Da Silva, and J.C. Moreno, Phys. Rev. A 48(6), 4682 (1993); E.E. Fill, Y. Li, G. Pretzler, D. Schlögl, J. Steingruber, and J. Nilsen, Physica Scripta 52, 158 (1995).

23. J.H. Scofield and B.J. MacGowan, Phys. Scr. 46, 361 (1992).

24. Y. Li et al, submitted for publication in Phys. Rev. A (1998).

25. G.J. Linford, E.R. Peressini, W.R. Sooy, and M.L. Spaeth, Appl. Opt. 13(2), 379 (1974).

26. J. Dunn et al, submitted to Opt. Lett. (1998).

27. J. Nilsen, J. Opt. Soc. Am. B 14, 1511 (1997).

28. J. Nilsen, Phys. Rev. A. 55, 3271 (1997); S.B. Healy et al, Opt. Commun. 132, 442 (1996); K.G. Whitney et al, Phys. Rev E 50, 468 (1994).

29. L.B. Da Silva, J.E. Trebes, R. Balhorn, S. Mrowka, E. Anderson, D.T. Attwood, T.W. Barbee Jr., J. Brase, M. Corzett, J. Gray, J.A. Koch, C. Lee, D. Kern, R.A. London, B.J. MacGowan, D.L. Matthews, and G. Stone, Science 258, 269 (1992).

30. L.B. Da Silva, T.W. Barbee Jr., R. Cauble, P. Celliers, D. Ciarlo, S. Libby, R.A. London, D. Matthews, S. Mrowka, J.C. Moreno, D. Ress, J.E. Trebes, A.S. Wan, and F. Weber, Phys. Rev. Lett. 74, 3991 (1995).

31. D.H. Kalantar, M.H. Key, L.B. Da Silva, S.G. Glendinning, J.P. Knauer, B.A. Remington, F. Weber, and S.V. Weber, Phys. Rev. Lett. 76, 3574 (1996).

32. G. Jamelot, F. Albert, A. Belsky, M. Boussoukaya, A. Carillon, A. Egbert, S. Hubert, P. Jaeglé, D. Joyeux, I. Kamenskikh, A. Klisnick, S. Meyer, D. Phalippou, D. Ros, B. Rus, S. Sebban, B. Wellegehausen, T. Woyvod, Ph. Zeitoun, and A. Zeitoun-Fakiris, SPIE Proceedings Vol. 3156, 124 (1997).

33. S. Basu, J.G. Goodberlet, M.H. Muendel, S. Kaushik, and P.L. Hagelstein, in X-ray Lasers 1992, ed. E. E. Fill, IOP Conf. Series No. 125, 71 (1992); S. Basu, P.L. Hagelstein, J.G. Goodberlet, M.H. Muendel, and S. Kaushik, App. Phys. B 57, 303 (1993). 
34. J. Dunn et al, submitted to Opt. Lett. (1998). 


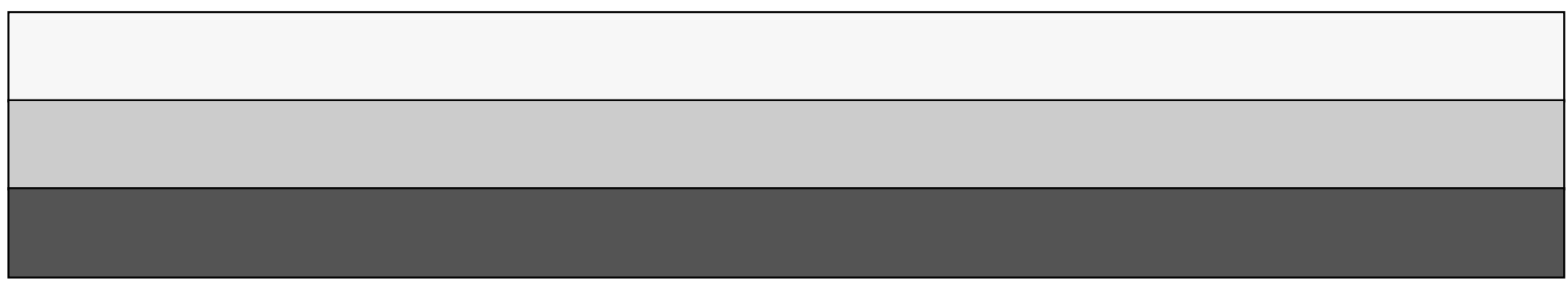

